\title{
Quasiparticle states and quantum interference induce by magnetic impurities on a two-dimensional topological superconductor
}

\author{
Zhen-Guo Fu, ${ }^{1,2}$ Ping Zhang, ${ }^{2, *}$ Zhigang Wang, ${ }^{2}$ and Shu-Shen Li $^{1}$ \\ ${ }^{1}$ State Key Laboratory for Superlattices and Microstructures, \\ Institute of Semiconductors, Chinese Academy of Sciences, \\ P. O. Box 912, Beijing 100083, People's Republic of China \\ ${ }^{2} L C P$, Institute of Applied Physics and Computational Mathematics, \\ P.O. Box 8009, Beijing 100088, People's Republic of China
}

\begin{abstract}
We theoretically study the effect of localized magnetic impurities on two-dimensional topological superconductor (TSC). We show that the local density of states (LDOS) can be tuned by the effective exchange field $m$, the chemical potential $\mu$ of TSC, and the distance $\Delta r$ as well as relative spin angle $\alpha$ between two impurities. The changes in $\Delta r$ between two impurities alter the interference and result in significant modifications to the bonding and antibonding states. Furthermore, the bound-state spin LDOS induced by single and double magnetic impurity scattering, the quantum corrals, and the quantum mirages are also discussed. Finally, we briefly compare the impurities in TSC with those in topological insulators.

PACS numbers: 73.20.-r, 72.10.Fk, 73.50.Bk, 74.90.+n
\end{abstract}

*zhang_ping@iapcm.ac.cn 


\section{INTRODUCTION}

The study of topological insulators (TI) has attracted considerable theoretical [1 [6] and experimental [7-11] interest over the past few years. These studies have opened a door for exploring the promising potential applications for spintronics with TI materials by their intrinsic strong spin-orbit coupling (SOC) nature. Very recently, a series of superconductor (SC)/TI hybrid structures, named as topological superconductors (TSCs) [12, 13], were proposed to realize Majorana fermions by using the proximity effect of SC and strong SOC of TI. Various transport proposals have been suggested to detect and manipulate the Majorana

fermions [14 20]. The experimental challenge along this line remains to be overcome. In another way Shindou et al. [21] suggested that the surface-adsorbed magnetic impurities can be effectively used to explore the Majorana fermions. Unfortunately, at present a detailed and revealed study of the magnetic impurity effects on the surface spectrum of TSC is still lacking, which is unexpected since a time reversal breaking perturbation is the most natural way to reveal both the topological and the superconducting properties of TSCs.

Motivated by the above-mentioned fact, in this paper we study the impurity states and impurity interference on the two-dimensional (2D) TSC, which are different from the ordinary SC systems because of the chirality and special energy spectrum of the itinerant electron in TSC. We show that there exist critical impurity-TSC spin coupling $m_{c}$ (following the appellation in Ref. [22]) and critical chemical potential $\mu_{c}$ for a single impurity, which correspond to unpaired zero-energy states. By analyzing the spin local density of states (LDOS), a quantum transition from the spin-unpolarized to the spin-polarized state is shown when the exchange coupling parameter exceeds $m_{c}$. A clear understanding of quantum interference is fundamental to our analysis of complex impurity structures. Therefore, we illustrate that the distance and the relative spin angle between two magnetic impurities can alter the quasiparticle interference effects, resulting in significant modifications to the bonding and antibonding states. For multiple impurities, we construct elliptical quantum corrals to study the spectral properties of quantum mirages and the influence of quantum corrals on the quantum interference effect between two magnetic impurities. We reveal that the nonmagnetic (antiferromagnetic) corral has strong (weak) influence. Finally, we briefly compare the impurity-TSC system with the impurity-TI system, and we find that for the latter, the intragap quasiparticle states can be arisen only when the scalar potential scatter- 
ing are taken into account. These findings, which can be detected by the scanning tunneling microscopy (STM) and scanning tunneling spectroscopy (STS) techniques, may be useful for determining the quasiparticle spectrum in TSC surface, and possess potential applications in quantum computation.

\section{THEORETICAL FORMALISM}

The 2D TSC by hybridizing an ordinary $s$-wave SC film with a TI film (say, for example, $\mathrm{Bi}_{2} \mathrm{Se}_{3}$ film) is described by the Bogoliubov-de Gennes Hamiltonian [12]

$$
H_{\mathrm{BdG}}(\boldsymbol{k})=\left(\begin{array}{cc}
h(\boldsymbol{k})-\mu & i \Delta \sigma_{y} \\
-i \Delta^{*} \sigma_{y} & -h^{*}(-\boldsymbol{k})+\mu
\end{array}\right),
$$

the basis for which is $\left(c_{\boldsymbol{k} \uparrow}, c_{\boldsymbol{k} \downarrow}, c_{-\boldsymbol{k} \uparrow}^{\dagger}, c_{-\boldsymbol{k} \downarrow}^{\dagger}\right)^{T}$. Here, for simplicity we just consider the strong SOC term

$$
h(\boldsymbol{k})=v_{F}\left(\sigma_{x} k_{x}+\sigma_{y} k_{y}\right) .
$$

In Eq. (1) $\mu$ is the chemical potential, $\sigma_{x, y}$ are Pauli matrices of electron spin, and the $s$-wave gap function is simply chosen to be a constant, $\Delta=\Delta_{0}$. The impurity potential can be expressed as

$$
V_{i}=\frac{1}{2}\left(U_{i} \sigma_{0}+J_{i} \boldsymbol{S}_{i} \cdot \boldsymbol{\sigma}\right) \tau_{z}
$$

where $\boldsymbol{S}_{i}=S \boldsymbol{n}_{i}$ is the classical spin (with its orientation vector $\boldsymbol{n}_{i}$ ) of the $i$ th magnetic impurity, $U_{i}$ and $J_{i}$ are the scalar potential and magnetic scattering strengths, and the Pauli matrix $\tau_{z}$ acts on the particle-hole space. We treat the impurity spin as a classical local effective exchange field $m_{i}=J_{i} S_{i} / 2$ under mean-field approximation [23]. By employing the non-self-consistent $T$-matrix method, we study the quantum states and interferences induced by the magnetic impurities on the TSC. The electronic Green's function in the presence of $N$ impurities is written as

$$
\begin{aligned}
G\left(\boldsymbol{r}, \boldsymbol{r}^{\prime}, i \omega\right) & =G_{0}\left(\boldsymbol{r}, \boldsymbol{r}^{\prime}, i \omega\right)+\sum_{i, j=1}^{N} G_{0}\left(\boldsymbol{r}, \boldsymbol{r}_{i}, i \omega\right) \\
& \times T\left(\boldsymbol{r}_{i}, \boldsymbol{r}_{j}, i \omega\right) G_{0}\left(\boldsymbol{r}_{j}, \boldsymbol{r}^{\prime}, i \omega\right),
\end{aligned}
$$

where the $T$-matrix is given by the Bethe-Salpeter equation

$$
T\left(\boldsymbol{r}_{i}, \boldsymbol{r}_{j}, i \omega\right)=V_{i} \delta_{i, j}+V_{i} \sum_{l=1}^{N} G_{0}\left(\boldsymbol{r}_{i}, \boldsymbol{r}_{l}, i \omega\right) T\left(\boldsymbol{r}_{l}, \boldsymbol{r}_{j}, i \omega\right)
$$


with the $4 \times 4$ materix $G_{0}\left(\boldsymbol{r}, \boldsymbol{r}^{\prime}, i \omega\right)$ obtained from the Fourier transformation of the unperturbed Green's function

$$
G_{0}(\boldsymbol{k}, i \omega)=\left[i \omega-H_{\mathrm{BdG}}\right]^{-1}
$$

for free TSC system. At half filling $\mu=0$, one can find an analytical expression of $G_{0}(\mathbf{r}=0, i \omega)$ for $|\omega|<\Delta_{0}$, written as

$$
\begin{aligned}
G_{0}(\mathbf{r}=0, i \omega) & =\frac{1}{4 \pi \varrho v_{F}^{2}} \ln \left(\frac{\Delta_{0}^{2}+\omega^{2}+v_{F}^{2} k_{c}^{2}}{\Delta_{0}^{2}+\omega^{2}}\right) \\
& \times\left(i \omega \tau_{0}+\Delta_{0} \tau_{2} \sigma_{2}\right),
\end{aligned}
$$

where $\varrho=1 / S_{\square}$ is the planar density with $S_{\square}$ the area of the STC surface, and $k_{c}$ is high momentum cutoff. Finally, we could get

$$
G_{0}(\mathbf{r}=0, \omega)=-A(\omega)\left(\omega \tau_{0}+\Delta_{0} \tau_{2} \sigma_{2}\right) / D,
$$

where $A(\omega)=\frac{2}{D} \ln \left(D / \sqrt{\Delta_{0}^{2}-\omega^{2}}\right)$ for $\Delta_{0} \ll D$ with $D=v_{F} k_{c}$. Here, we have used $4 \pi \varrho v_{F}^{2}=D^{2}$ with $\varrho=k_{c}^{2} / 4 \pi$.

The total Green's function $G\left(\boldsymbol{r}, \boldsymbol{r}^{\prime}, \omega\right)$ in Eq. (4) can be rewritten as

$$
G\left(\boldsymbol{r}, \boldsymbol{r}^{\prime}, \omega\right)=G_{0}\left(\boldsymbol{r}, \boldsymbol{r}^{\prime}, \omega\right)+\mathcal{G}_{0} \mathcal{T} \mathcal{G}_{0}^{\prime}
$$

where the $4 \times 4 N$ matrix

$$
\mathcal{G}_{0}=\left(G_{0}\left(\boldsymbol{r}, \boldsymbol{r}_{1}, \omega\right) \cdots \cdots G_{0}\left(\boldsymbol{r}, \boldsymbol{r}_{N}, \omega\right)\right)
$$

and the $4 N \times 4$ matrix

$$
\mathcal{G}_{0}^{\prime}=\left(G_{0}\left(\boldsymbol{r}_{1}, \boldsymbol{r}^{\prime}, \omega\right) \cdots \cdots G_{0}\left(\boldsymbol{r}_{N}, \boldsymbol{r}^{\prime}, \omega\right)\right)^{T},
$$

denote the propagation of electrons from the STM tip to the impurities as well as from the impurities to the STM tip. Here, the $4 N \times 4 N$ matrix $\mathcal{T}$ in Eq. (9) is a rewritten form of the Eq. (5) for the T-matrix,

$$
\mathcal{T}=\mathcal{H}_{\text {imp }}+\mathcal{H}_{\text {imp }} \mathcal{G}_{0} \mathcal{T}
$$

with the impurity Hamiltonian

$$
\mathcal{H}_{i m p}=\left(\begin{array}{cccc}
V_{1} & \mathbf{0} & \cdots & \mathbf{0} \\
\mathbf{0} & V_{2} & \cdots & \mathbf{0} \\
\vdots & \vdots & \ddots & \vdots \\
\mathbf{0} & \mathbf{0} & \cdots & V_{N}
\end{array}\right)
$$


containing all of the impurity scattering potentials $V_{i}(i=1, \cdots, N)$. The information about the propagation between the impurities are included in $\mathcal{G}_{0}$, which can be expressed as

$$
\mathbf{G}_{0}=\left(\begin{array}{cccc}
G_{0}\left(\boldsymbol{r}_{1}, \boldsymbol{r}_{1}\right) & G_{0}\left(\boldsymbol{r}_{1}, \boldsymbol{r}_{2}\right) & \cdots & G_{0}\left(\boldsymbol{r}_{1}, \boldsymbol{r}_{N}\right) \\
G_{0}\left(\boldsymbol{r}_{2}, \boldsymbol{r}_{1}\right) & G_{0}\left(\boldsymbol{r}_{2}, \boldsymbol{r}_{2}\right) & \cdots & G_{0}\left(\boldsymbol{r}_{2}, \boldsymbol{r}_{N}\right) \\
\vdots & \vdots & \ddots & \vdots \\
G_{0}\left(\boldsymbol{r}_{N}, \boldsymbol{r}_{1}\right) & G_{0}\left(\boldsymbol{r}_{N}, \boldsymbol{r}_{2}\right) & \cdots & G_{0}\left(\boldsymbol{r}_{N}, \boldsymbol{r}_{N}\right)
\end{array}\right) .
$$

As a result, one can obtain

$$
\mathcal{T}=\frac{\mathcal{H}_{i m p}}{I-\mathcal{H}_{i m p} \mathbf{G}_{0}}
$$

where $I$ is a $4 N \times 4 N$ unity matrix. Finally, by using these equations, one can easily finish the numerical calculations with $N$ impurities, and then get the $\operatorname{LDOS} \rho(\mathbf{r}, E)$ of TSC with impurities, which is given by

$$
\rho(\boldsymbol{r}, \omega)=-\frac{1}{\pi} \operatorname{Im} \sum_{i=1}^{2} G_{i i}(\boldsymbol{r}, \boldsymbol{r}, \omega) \equiv-\frac{1}{\pi} \operatorname{Im} \operatorname{Tr} G^{(p)}(\boldsymbol{r}, \boldsymbol{r}, \omega),
$$

where $G^{(p)}\left(\boldsymbol{r}, \boldsymbol{r}^{\prime}, \omega\right)$ is the particle Green function. Furthermore, the spin LDOS is written as

$$
\boldsymbol{s}(\boldsymbol{r})=-\frac{1}{2 \pi} \operatorname{Im} \operatorname{Tr}\left[G^{(p)}(\boldsymbol{r}, \boldsymbol{r}, \omega) \boldsymbol{\sigma}\right] .
$$

For definiteness we set $k_{c}=0.3 \sim 0.4 \AA^{-1}, v_{F}=2.55 \mathrm{eV} \cdot \AA$, and $\Delta_{0}=1 \mathrm{meV}$, which give out

a coherence length $\xi=v_{F} / \Delta_{0}=255 \mathrm{~nm}$. The lattice constance is chosen as $a_{0}=4.25 \AA$, and $0^{+}=0.02 \mathrm{meV}$ in following calculations. Here the chosen value of $\Delta_{0}$ is consistent with recent theoretical prediction [12] and experimental implication [24] on $\mathrm{Cu}_{x} \mathrm{Bi}_{2} \mathrm{Se}_{3}$.

\section{RESULTS AND DISCUSSIONS}

\section{A. Single magnetic impurity}

Similar to the ordinary $s$-wave SC systems, the purely scalar potential scattering cannot form impurity bound state in the gap of TSC. Therefore, we focus on the magnetic scattering effects in the following calculations. Firstly, we consider a single magnetic impurity in STC.

We know that the $T$-matrix possesses poles at resonance frequencies $\omega_{\text {res }}^{(1,2)}$, which can reflect the presence of bound states. The spectroscopic evidences for bound states are a pair of peaks in the LDOS, which changes with $m$ as well as $\mu$ of the impurity-TSC system. As 


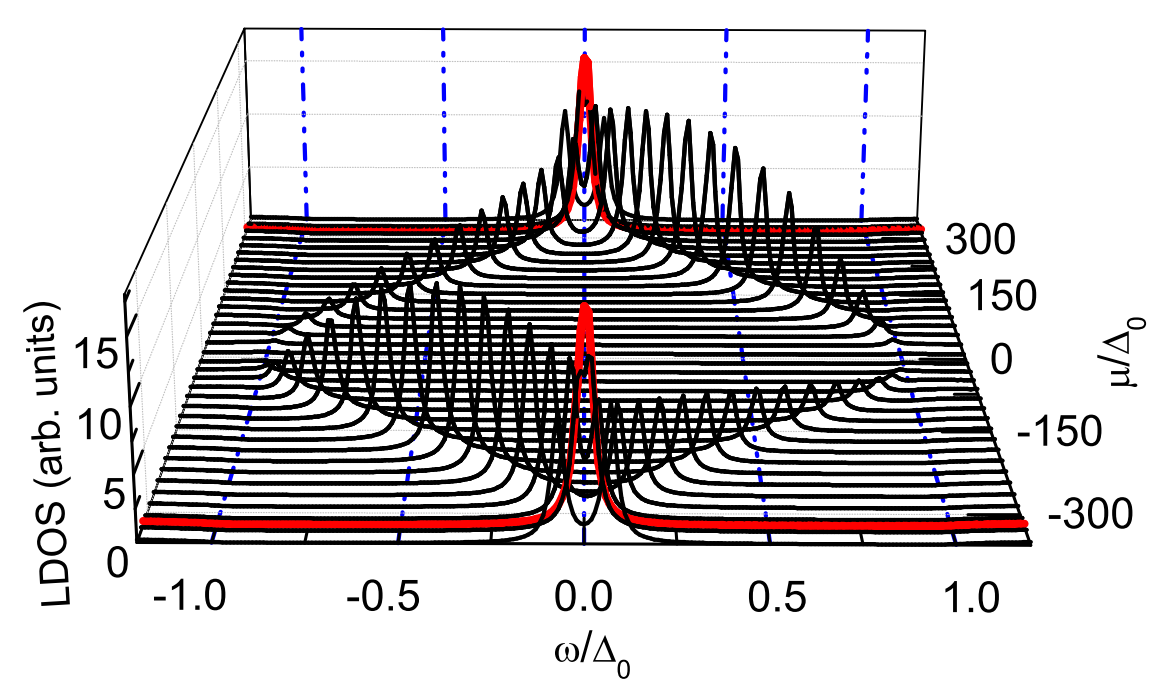

FIG. 1: (Color online) The LDOS $\rho(\boldsymbol{r}=\mathbf{0}, \omega)$ of a single impurity located at origin with $m=0.8$ $\mathrm{eV}, \boldsymbol{S} \| \hat{z}$, and $U=0$, as a function of frequency $\omega$ for a series of chemical potential $\mu$ in a TSC. The unpaired zero-energy state are illustrated by red curves as $\mu$ increases to $\mu_{c} \approx 0.32 \mathrm{eV}$.

$m$ increases, $\omega_{r e s}^{(1,2)}$ approach the chemical potential, and at the critical coupling $m_{c}, \omega_{r e s}^{(1,2)}$ become to be degenerate at the zero energy. If $|\omega|$ is not close to $\Delta_{0}$, one have a reasonable approximation $A(\omega) \approx A(0)$, and then from the Green's function $G_{0}(\boldsymbol{r}=0, \omega)$ in Eq. (7), we can easily obtain the $T$-matrix at half filling $\mu=0$, written as

$$
T(\omega)=\left[V^{-1}-G_{0}(\boldsymbol{r}=0, \omega)\right]^{-1}
$$

for a single impurity case, which gives out critical coupling

$$
m_{c}(U) \simeq \frac{1}{2} \sqrt{4 D^{2} /\left(\Delta_{0} A(0)\right)^{2}+U^{2}}
$$

and the bound-state energies

$$
\omega_{r e s}^{(1,2)}=-2 c_{m} \pm \sqrt{c_{u}^{2}+\Delta_{0}^{2}}
$$

for $m<m_{c}(U)$, where $c_{m}=2 D m /\left[A(0)\left(4 m^{2}-U^{2}\right)\right]$ and $c_{u}=2 D U /\left[A(0)\left(4 m^{2}-U^{2}\right)\right]$. It is clear from Eq. (19) that taking into account the scalar potential $U$ in the impurity potential can effectively increase the critical coupling $m_{c}(U)$.

In the following calculations of $\mu \neq 0$, for briefness we ignore the influence of the scalar potential term $(U=0)$. From numerical calculations, we find that the critical magnetic moment 
$m_{c}=3.0,1.15,0.98$, and $0.71 \mathrm{eV}$ for $U=0$ corresponding to the chemical potential $\mu=0.05$, $0.2,0.25$, and $0.37 \mathrm{eV}$ respectively. On the other hand, in Fig. 1 we present the LDOS at the impurity site $\boldsymbol{r}=(0,0)$ as a function of $\mu$ for a fixed exchange field $m=0.8 \mathrm{eV}$. One can see that the impurity resonance phenomenon approaches to vanish when tuning the chemical potential to half filling $(\mu=0)$. The observable intragap resonant states with energies of $\omega_{\text {res }}^{(1,2)}$ evolve from non-zero $\mu$, and their energy difference $\left(\left|\omega_{r e s}^{(2)}-\omega_{r e s}^{(1)}\right|\right)$ decreases with increasing $\mu$. Particularly, at a critical value $\mu_{c}$ of the chemical potential, the particle- and hole-like bound states are degenerate at zero energy with equal spectral weight. In Fig. 1 we determine this critical chemical potential to be $\mu_{c}= \pm 320 \Delta_{0}$ for the occurrence of zero-energy bound states (see the red curves). Therefore, we can conclude that by tuning the spin coupling or the host's chemical potential, the zero-energy bound states could be detected on 2D TSC by employing spectral techniques such as STS.

The occurrence of zero-energy bound states is a result of competition among the pairingcondensation energy, the magnetic interaction, and strong SOC. At these critical points, the ground state of STC may become thermodynamically unstable, arising the quantum transformation of system from spin-unpolarized to the spin-polarized state. As regarding to the spin polarization, one should consider the spin LDOS around the impurity, from which the spatially resolved spin polarization can be obtained by performing integral over energy,

$$
\langle\boldsymbol{s}(\boldsymbol{r})\rangle=-\frac{1}{\pi} \int_{-\infty}^{0} d \omega \boldsymbol{s}(\boldsymbol{r}, \omega),
$$

as well as the total spin polarization $\langle\boldsymbol{s}\rangle=\sum_{\boldsymbol{r}} \boldsymbol{s}(\boldsymbol{r})$. The typical spatial distribution of spin LDOS is plotted in Fig. 2 at resonance frequency $\omega_{r e s}^{(1)}=-0.38 \Delta_{0}$ for two choices of spin orientations of the single magnetic impurity. Figure 2(a) is the case that the local moment $\boldsymbol{S} \| \hat{z}$, while Fig. 2(b) corresponds to $\boldsymbol{S} \| \hat{x}$. It is clear that the impurity induces not only a $z$-direction spin polarization which decays with oscillations, but also an $x y$-plane spin polarization in the present TSC system. The spin LDOS possesses rotation symmetry about $z$-axis for a $z$-direction impurity spin, shown as Fig. 2(a). However, the rotation symmetry is broken when the impurity spin lies in plane. We find from Fig. 2(b), for example, that the in-plane component $s_{\|}(\boldsymbol{r}, \omega)$ rotates anticlockwise (clockwise) in the upper (lower) halfplane. These results can be understood by the effective magnetic field $\boldsymbol{B}_{\text {eff }}=v_{F} \boldsymbol{k}$ induced by the strong SOC in the 2D TSC, which is similar to the case of a magnetic impurity on TI surface [23]. Because it is locked to the momentum of electron, with the moving of electron, 


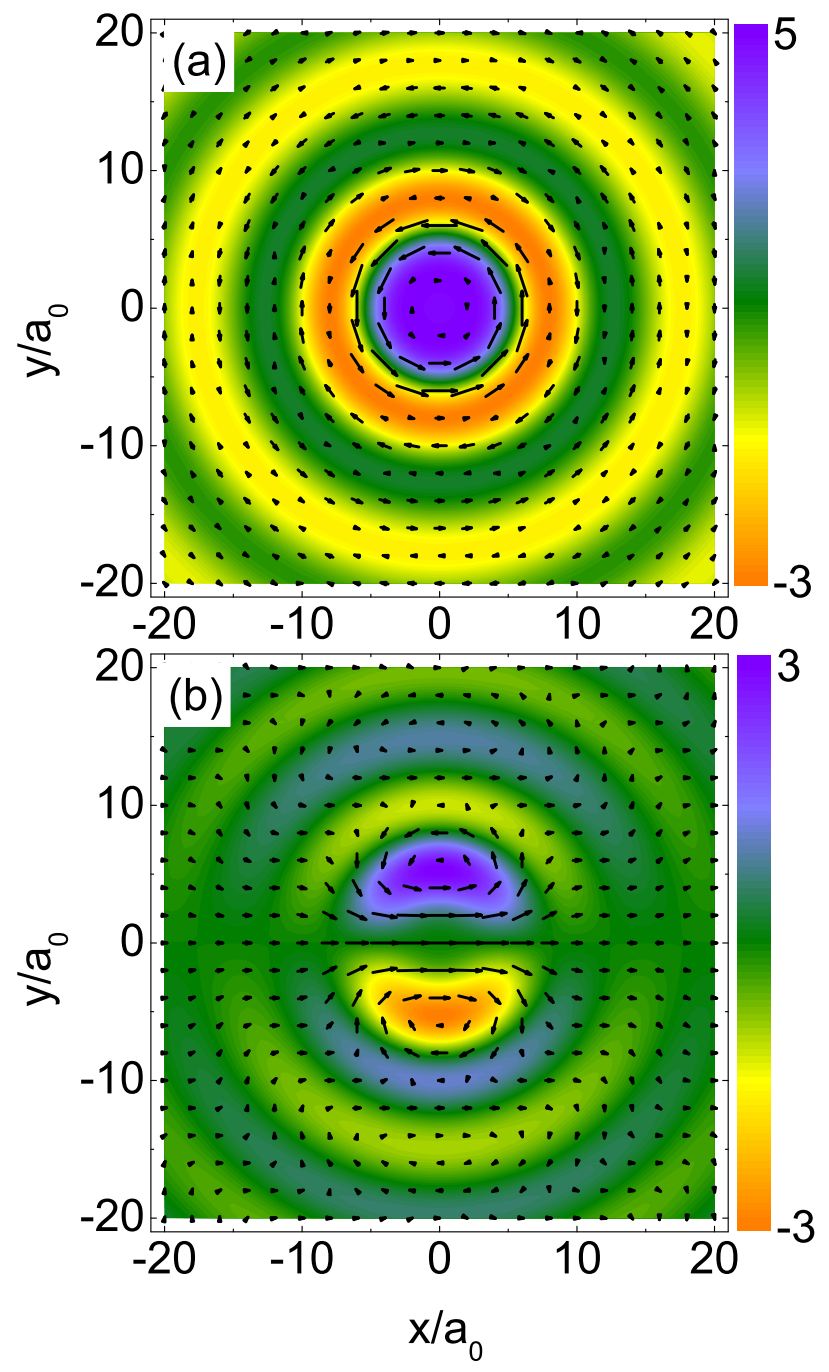

FIG. 2: (Color online) The spatial distribution of spin LDOS at resonance frequency $\omega_{r e s}=-$ $0.38 \Delta_{0}$ for a single magnetic impurity located at origin with $z$-direction (a) and $x$-direction polarization. The background color denotes the $z$ component of spin LDOS $s_{z}(\boldsymbol{r}, \omega)$ while the arrow indicates the $x y$-plane component $s_{\|}(\boldsymbol{r}, \omega)$. The parameters are chosen as $m=0.8 \mathrm{eV}$ and $\mu=0.2$ $\mathrm{eV}$.

the spin undergoes a gyroscopic precession in the plane of perpendicular to the propagation orientation, which arises corresponding slant of spin LDOS $\boldsymbol{s}(\boldsymbol{r}, \omega)$.

Taking a further step, corresponding to Fig. 2(a), we find that the total spin polarization $\left\langle s_{z}\right\rangle \approx 0.04<1 / 2$, which indicates a negligibly small spin polarization. This is consistent with our choice of $m$, which is lower than $m_{c}$; when $m>m_{c}$, an obvious spin polarization is observed. As a result, the resonance peaks at negative (positive) energy region possesses 
a particle-like and hole-like spin-chiral states $|p, \nearrow\rangle$ and $|h, \searrow\rangle$, respectively, where $\nearrow$ and $\searrow$ represent two different spin chiralities. This is different from that on the ordinary $s$ wave SC surface with classical magnetic impurity, where the particle/hole spin is parallel or antiparallel to the impurity spin $S[22,25,26]$. Notice that we have not found a quantum transformation from spin-unpolarized to the spin-polarized state by increasing $\mu$.

\section{B. Two magnetic impurities}

When the two impurities are close to each other, electrons will be scattered by both impurities, resulting in quantum interference of electronic waves. The interference effect is related to the scattering strength, the distance as well as spin angle between two impurities. There are two important consequences occurred due to the interference effect: (i) One is that the interference can change the formation of the so-called bonding and antibonding states $|p(h)\rangle_{b, a}=(|p(h), 1\rangle \pm|p(h), 2\rangle) / \sqrt{2}$ for the particle-like (hole-like) states, with $|p, i=1,2\rangle$ and $|h, i=1,2\rangle$ being the bound states of each impurity; (ii) The other one is that the spin polarization will be altered too, which can be observed from the spin LDOS patterns.

Here, we focus on two identical magnetic impurities located at $\boldsymbol{r}_{1}=(0,0)$ and $\boldsymbol{r}_{2}=(\Delta r, 0)$ with the same exchange field $m=0.8 \mathrm{eV}$. Firstly, the LDOS for two impurities located at $\boldsymbol{r}_{1}=(0,0)$ and $\boldsymbol{r}_{2}=\left(8 a_{0}, 0\right)$ is shown by the red curve in Fig. 3(a), which exhibits four

resonance peaks with peaks $\Omega_{i=1,2}$ corresponding to the particle-like states $|p\rangle_{b, a}$ and peaks $\Omega_{i=3,4}$ to the hole-like states $|h\rangle_{b, a}$. If the interference of $|p, 1\rangle$ and $|p, 2\rangle$ is constructive (destructive) between two impurities, the bonding state $|p / h\rangle_{b}\left(|p / h\rangle_{a}\right)$ is formed, which can be observed from the spatial LDOS patterns. For this purpose, we plot in Figs. 3(b) and 3(c) the spatial LDOS corresponding to the particle-like state peaks $\Omega_{1}=-0.74 \Delta_{0}$ and $\Omega_{2}=-0.27 \Delta_{0}$ respectively (the positions of two impurities are indicated by black dots). It is obvious from Fig. 3(b) that the LDOS are located in the middle region between two impurities and reach maximum at $\Delta r=\left(4 a_{0}, 0\right)$, therefore, peak $\Omega_{1}$ corresponds to a bounding state $|p\rangle_{b}$. On the other hand, peak $\Omega_{2}$ should correspond to an antibounding state $|p\rangle_{a}$ since the LDOS vanishes at middle point between two impurities, see Fig. 3(c).

However, the interference pattern between the bonding and antibonding states changes by varying the distance between the two impurities, i.e., the parity and resonance energy of the two-impurity states oscillate with increasing impurity separation. This can be observed from 

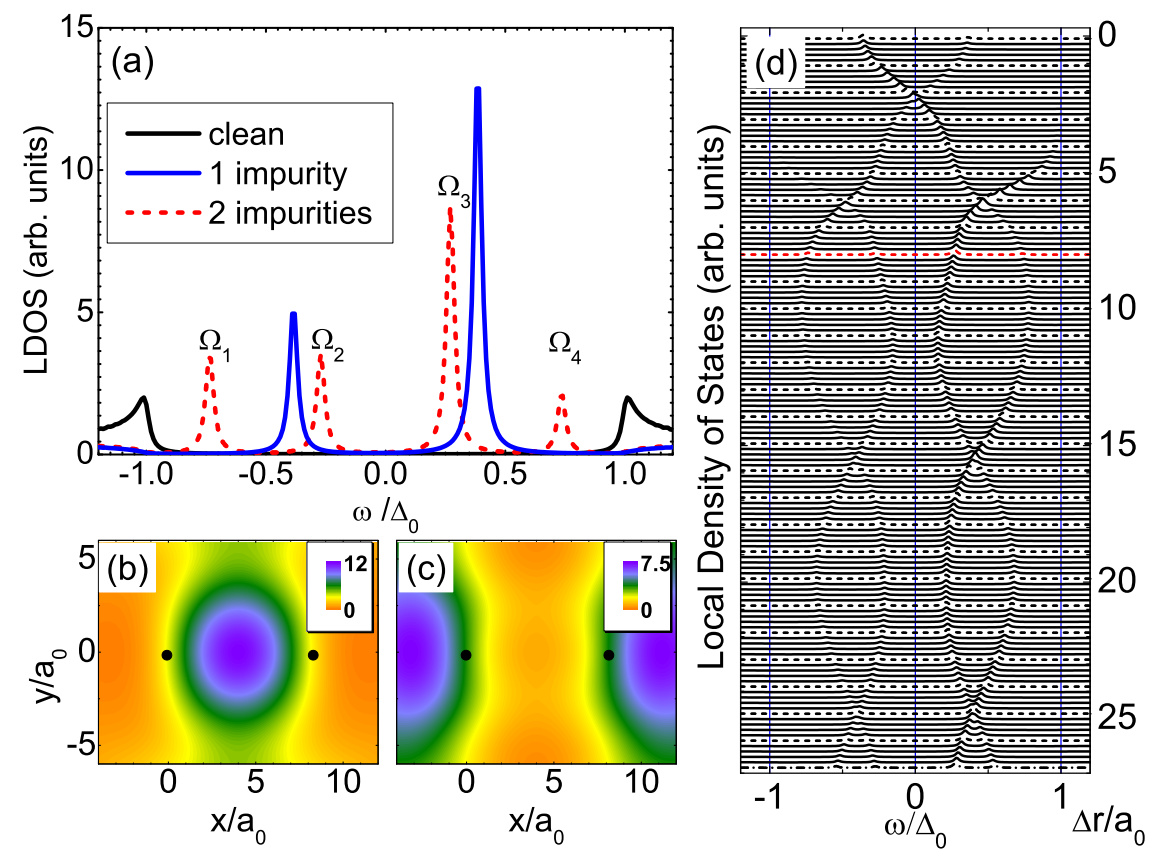

FIG. 3: (Color online) (a) LDOS at impurity site for the clean surface (black curve), a single impurity (blue curve), and two impurities (red dotted curve) located at $\boldsymbol{r}_{1}=(0,0)$ and $\boldsymbol{r}_{2}=\left(8 a_{0}, 0\right)$ with parallel spins along $z$-direction, respectively. (b) and (c) Spatial dependence of LDOS corresponding to peaks $\Omega_{1}$ and $\Omega_{2}$ in (a), respectively. The locations of impurities are denoted by black dots. (d) LDOS at $\boldsymbol{r}=\mathbf{0}$ as a function of $\Delta r$ for two identical impurities with parallel spins. The red dotted curve is for $\Delta r=8 a_{0}$.

Fig. 3(d), which shows the two-impurity distance $\Delta r$ dependency of LDOS. For instance, $|p\rangle_{b}$ corresponds to peak $\Omega_{2}$ while $|p\rangle_{a}$ corresponds to peak $\Omega_{1}$ when $\Delta r=5 a_{0}$ or $20 a_{0}$. The curves in Fig. 3(d) are moved in order to illustrate the finer and more clear resonance frequencies. The frequencies of the resonance oscillations, and at the same time the amplitude of LDOS as well as their energy width changes. Moreover, we find that there exists a critical distance $\Delta r_{c} \approx 2 a_{0}$ similar to the critical exchange parameter $m_{c}$ and critical chemical potential $\mu_{c}$ for single impurity case. At this point, the bound state energy of particle-like $|p\rangle_{b}$ (hole-like $\left.|h\rangle_{b}\right)$ crosses zero, and the state transforms into hole-like $|h\rangle_{b}$ (particle-like $|p\rangle_{b}$ ). Similar result has been theoretically observed in the ordinary $s$-wave SC materials [25]. Whether or not a quantum spin polarization transition occurs with increasing $\Delta r$ to exceed $\Delta r_{c}$ is not clear, and further work are under way to study this interesting issue. Besides, it is obvious that when the distance of two impurities is as small as $\Delta r \lesssim 4 a_{0}$, only two intragap bound 

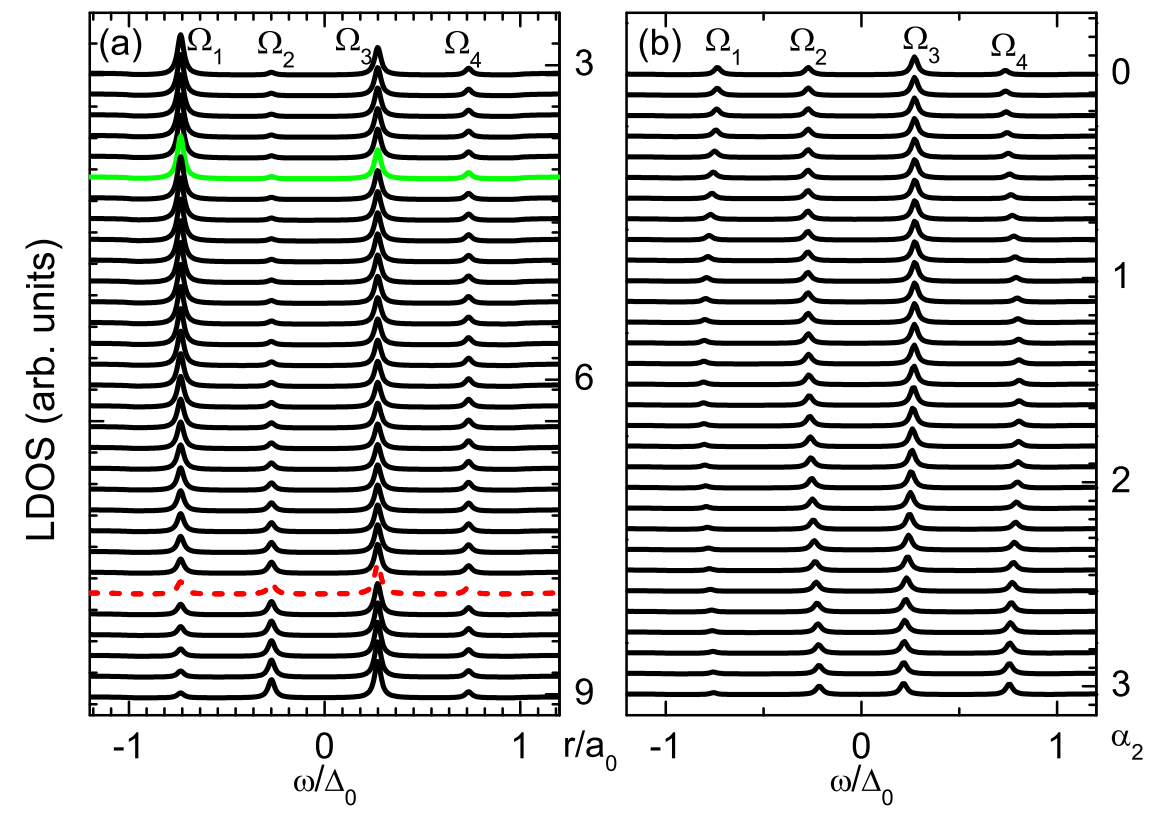

FIG. 4: (Color online) (a) LDOS as a function of spatial position $\boldsymbol{r}=(r, 0)$ for two impurities apart $8 a_{0}$ with parallel spins $S \hat{z}$. The red dashed curve represents the LDOS at $\boldsymbol{r}_{2}$, and the green curve is for the midpoint $\boldsymbol{r}=\left(4 a_{0}, 0\right)$. (b) LDOS at $\boldsymbol{r}=\mathbf{0}$ as a function of the angle $\alpha_{2}$ between two impurity spins. The parameters are chosen as $m=0.8 \mathrm{eV}$ and $U=0$.

states are found, while there appears four non-degenerate bound states with increasing $\Delta r$ to be larger than $4 a_{0}$. With further increasing $\Delta r$, the interference effect during the electron scattering processes by both impurities gets so weak that the bound states become degenerate.

An additional result of quantum interference between two impurities is the change with the position of STM tip $\boldsymbol{r}$ in the number of observable low-energy resonance peaks. For illustration we present in Fig. 4(a) the LDOS as a function of $\boldsymbol{r}=(r, 0)$ for two impurities with parallel spins located at $\boldsymbol{r}_{1}=(0,0)$ and $\boldsymbol{r}_{2}=\left(8 a_{0}, 0\right)$. From four main low-energy intragap resonances $\Omega_{i=1, \cdots, 4}$, one can find that the resonance amplitude of particle-like (hole-like) peaks $\Omega_{1}$ and $\Omega_{2}\left(\Omega_{3}\right.$ and $\left.\Omega_{4}\right)$ varies obviously (unobviously) with $\boldsymbol{r}$. For example, for the uppermost curve $\boldsymbol{r}=\left(3 a_{0}, 0\right)$, the peak $\Omega_{1}\left(\Omega_{2}\right)$ is sharp (smooth), while it is weaker (stronger) when the tip moves to $\boldsymbol{r}=\left(9 a_{0}, 0\right)$, see the bottom curve in Fig. 4(a). This is consistent with Fig. 3(b) because $\Omega_{1}$ corresponds to a bonding state $|p\rangle_{b}$. As mentioned above, the quantum interference effect could also be tuned through varying the angle $\alpha_{2}$ 


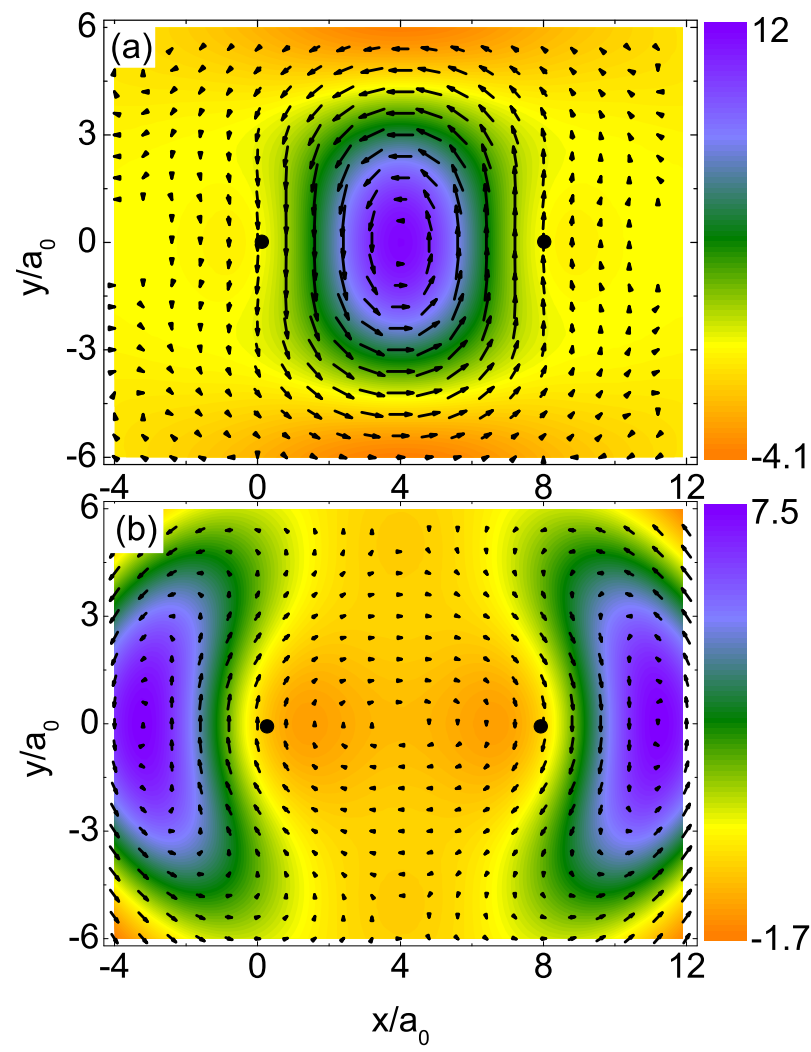

FIG. 5: (Color online) Spin LDOS at resonance energy $\Omega_{1}$ (a) and $\Omega_{2}$ (b) for two impurities. The parameters are the same as those in Fig. 3.

between two impurity spins, as shown in Fig. 4(b) for two impurities apart $8 a_{0}$. We find that the resonance frequencies $\Omega_{i}$ change with $\alpha_{2}$.

We also investigate the spin LDOS interference patterns of two impurities apart $8 a_{0}$ with parallel spins in the $z$-direction, and the results are presented in Fig. 5, It is found from Fig. [5(a) [5(b)] that on one hand, at the midpoint $\Delta r / 2$ between the two impurities, the $z$-component $s_{z}(\boldsymbol{r}, \omega)$ reaches its maximal (minimal) value, while the in-plane component $s_{\|}(\boldsymbol{r}, \omega)$ vanishes when the bonding (antibonding) state is formed. Thus the spin in the bonding (antibonding) particle-like state $|p\rangle_{b}\left(|p\rangle_{a}\right)$ is completely parallel (antiparallel) at $\Delta r / 2$ to the impurity spins. On the other hand, the in-plane component $s_{\|}(\boldsymbol{r}, \omega)$ rotates anticlockwise (clockwise) between the two impurities when the bonding (antibonding) state is formed. These findings may contribute to potential applications in the spin selection and also may be useful for analyzing the bound-state electron mediated Ruderman-KittelKasuya-Yosida (RKKY) spin-spin interaction between magnetic impurities in TSC. 


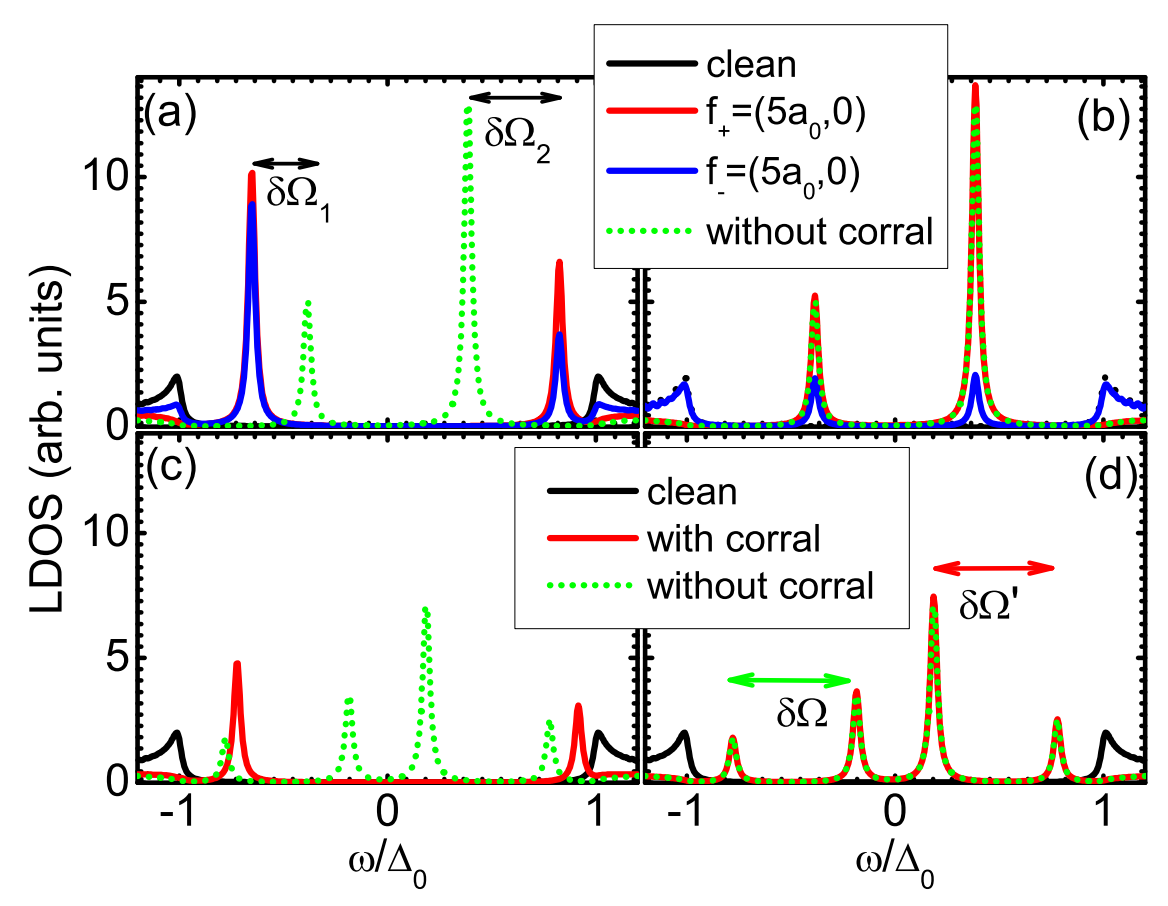

FIG. 6: (Color online) (a-b): LDOS at the occupied focus $f_{+}$(red curve) and empty focus $f_{-}$ (blue curve) for a nonmagnetic $U=0.8 \mathrm{eV}$ (a) and antiferromagnetic $m=0.8 \mathrm{eV}$ (b) corral with a magnetic impurity located at $f_{+} \cdot(\mathrm{c}-\mathrm{d})$ : Splitting of the resonance peaks in LDOS at $f_{+}$with (red curves) and without (green dotted curves) a nonmagnetic corral (c) and a antiferromagnetic corral (d) respectively, for two magnetic impurities with parallel spins. The chemical potential is $\mu=0.2$ $\mathrm{eV}$.

\section{Multiple impurities - quantum corral}

The nanostructrues, such as quantum corrals constructed by impurities on STC, is also an issue of importance since different impurity structures usually lead to different quantum interference behaviors. Here, as an illustrative example, we briefly consider the spectral property of elliptical quantum corrals with semimaxes $a=10 a_{0}, b=8.66 a_{0}$, and foci $f_{ \pm}=\left( \pm 5 a_{0}, 0\right)$, which are constructed by 30 nonmagnetic or magnetic impurities on STC. In the following, we fix $\mu=0.2 \mathrm{eV}$ and define $U_{i}=0.8 \mathrm{eV}$ for scalar impurities $\left(J_{i}=0\right)$, while $m_{i}=0.8 \mathrm{eV}\left(U_{i}=0\right)$ for magnetic ones.

Firstly, we consider the quantum mirage effect of a magnetic impurity that is projected from the occupied into the empty focus of elliptical corrals. The numerical spectral property of mirage versus the energy $\omega$ are presented in Figs. 6(a) and 6(b) for nonmagnetic and 
antiferromagnetic quantum corrals, respectively. The resonance peaks $\Omega_{1,2}$ of the mirage at empty focus $f_{-}$(blue curve) are in good agreement with those at occupied focus $f_{+}$(red curve), i.e., the mirage is clear. Comparing with the case without corral, we find that the nonmagnetic corral unsymmetrically shifts the particle-like and hole-like peaks. In Fig. 6(a) the particle-like peak is shifted down by $\delta \Omega_{1}=0.27 \Delta_{0}$, while the hole-like peak is shifted up by $\delta \Omega_{2}=0.44 \Delta_{0}$. Surprisingly, although the mirage is obvious in antiferromagnetic corral [Fig. 6(b)], the resonance peaks without corral are not shifted by antiferromagnetic one in 2D TSC.

Furthermore, the influence of quantum corrals on the quantum interference effects between two spin-parallel magnetic impurities apart $2\left|f_{+}\right|$on the TSC surface are also discussed. Although the interference is dependent on many physical factors, such as spatial locations, impurities scattering strength, relative angle between two impurity spins and so on, for briefness we only consider the energy dependency of LDOS at $f_{+}$of corrals. The results are shown in Figs. 6(c) and 6(d) for nonmagnetic and antiferromagnetic corrals, respectively. One can find that the influence of a nonmagnetic corral is prominent, see the red (green dotted) curves in Figs. 6(c) which correspond to the presence (absence) of corrals. However, the antiferromagnetic corral represents weak influence (the splitting of bound state peaks is almost the same as that without corral, see Fig. $6(\mathrm{~d}))$. This result is different from

that in ordinary SC system [25]. We also find that the influence of corrals can be changed by tuning the host chemical potential. When $\mu=0.25 \mathrm{eV}$, for example, in the absence of corral the splitting of peaks is $\delta \Omega / \Delta_{0}=0.595$, while it increases to $\delta \Omega^{\prime} / \Delta_{0}=0.65$ in the presence of an antiferromagnetic corral.

\section{COMPARISON WITH IMPURITY-TI SYSTEM}

Before ending this paper, we would like to qualitatively compare the magnetic impurities on TSC with those on TI surface. For briefness, we just consider one- and two-impurity cases. In the presence of magnetic impurities, the TI surface is described by

$$
\begin{aligned}
H & =h(\boldsymbol{k})+\mu \sigma_{0}+\Delta_{0} \sigma_{z} \\
& +\sum_{i=1}^{N}\left[U \sigma_{0}+J_{i} S / 2 \sigma_{z}\right] \delta\left(\boldsymbol{r}-\boldsymbol{r}_{i}\right),
\end{aligned}
$$




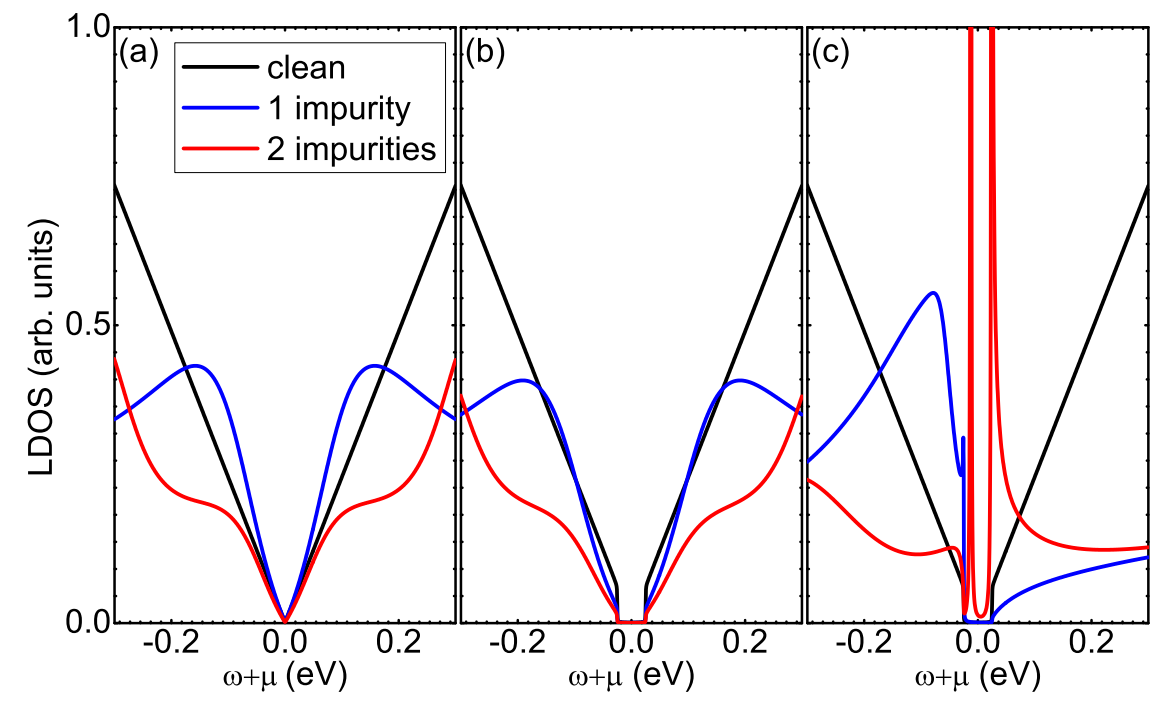

FIG. 7: (Color online) (a) LDOS at impurity site $\boldsymbol{r}_{1}=(0,0)$ on gapless (a) and gapped $\Delta_{0}=25$ meV (b) and (c) topological insulator surface. The black, blue, and red curves correspond to clean surface, one single impurity, and two impurities apart $2 a_{0}$, respectively. In (a) and (b) $U=0$, while in (c) $U=2 \mathrm{eV}$. The magnetic moment is chosen as $m=0.8 \mathrm{eV}$.

where $2 \Delta_{0}$ in the third term is the energy gap induced by the effective mass of Dirac fermions. The eigenvalues of the free part of Eq. (22) are

$$
\epsilon_{k}= \pm \sqrt{\left(v_{F} k\right)^{2}+\Delta_{0}^{2}}+\mu
$$

which indicates that the chemical potential is not important for impurity scattering in TI since it just move the levels up and down.

The numerically calculated LDOS at impurity site $\boldsymbol{r}_{1}=(0,0)$ for TI surface with $\mu=0.2$ eV are shown in Fig. 7, in which the black curves are for clean surface, and the blue (red) curves are for TI surface with one (two) impurity. In Figs. 77(a) and 7(b), we set the scalar scattering potential $U=0$, while $U \neq 0$ in Fig. 7(c). One can see that the low-energy LDOS is reshaped prominently by the impurity scattering on massless as well as massive TI surface. For example, in Fig. 7(a) the resonance peaks (blue curve) induced by one impurity are smoothed (blue curve) due to the interference between two impurities. Similar to the impurity on STC surface, the particle-hole symmetry holds on if we ignore $U$; Differing from the case of TSC surface, however, we find that the magnetic impurity on TI surface does not introduce intragap resonance peaks if one ignores $U$, see Fig. 7 (b). When the scalar potential 
is taken into account, we find that, on one hand, the scalar potential introduces intragap resonance peaks, which differs from the TSC case. On the other hand, the particle-hole symmetry is now broken, as shown in Fig. 7(c). These resonance states could be resolved from STM measurements, and thereby we hope the different impurity effects in TSC and TI materials could be found in experiment.

\section{CONCLUSION}

In summary, we have studied the spectral properties of quasiparticle states induced by localized classical magnetic impurities on 2D TSC. For the single impurity case, the critical exchange field $m_{c}$ and critical chemical potential $\mu_{c}$ are determined, and moreover, the spin LDOS is discussed. Spin polarization transformation has been found in TSC system when $m>m_{c}$. For two-impurity scattering, we have discussed the interference effects by changing the distance and relative spin angle between two impurities. We have showed that the bound states can be changed by varying the distance between the two impurities. The quantum mirages and the influence of quantum corrals on the quantum interference effect between two impurities have also been analyzed. The nonmagnetic corral exhibits stronger influence than the antiferromagnetic one. Finally, we briefly compare the impurities in TSC with those in TI. The results illustrated that the intragap resonance states could be found in TI so long as the scalar scattering potential is taken into account. These predictions, which could be observed by STM and STS techniques, may be useful for exploring the electronic structures of TSC as well as TI materials.

This work was supported by NSFC under Grants No. 90921003, No. 60776063, No. 60821061, and 60776061, and by the National Basic Research Program of China (973 Program) under Grants No. 2009CB929103 and No. G2009CB929300.

[1] C. L. Kane and E. J. Mele, Phys. Rev. Lett. 95, 146802 (2005).

[2] B. A. Bernevig, T. L. Hughes, and S.-C. Zhang, Science 314, 1757 (2006).

[3] L. Fu, C. L. Kane, and E. J. Mele, Phys. Rev. Lett 98, 106803 (2007).

[4] J. E. Moore and L. Balents, Phys. Rev. B 75, 121306(R) (2007).

[5] X.-L. Qi, T. L. Hughes, and S.-C. Zhang, Phys. Rev. B 78, 195424 (2008). 
[6] H. Zhang, C.-X. Liu, X.-L. Qi, X. Dai, Z. Fang and S.-C. Zhang, Nat. Phys. 5, 438 (2009).

[7] M. König, S. Wiedmann, C. Brüne, A. Roth, H. Buhmann, L. W. Molenkamp, X.-L. Qi, S.-C. Zhang, Science 318, 766 (2007).

[8] D. Hsieh, D. Qian, L. Wray, Y. Xia, Y. S. Hor, R. J. Cava and M. Z. Hasan, Nature 452, 970 (2008); D. Hsieh, Y. Xia, D. Qian, L. Wray, F. Meier, J. H. Dil, J. Osterwalder, L. Patthey, A. V. Fedorov, H. Lin, A. Bansill, D. Grauer, Y. S. Hor, R. J. Cava, and M. Z. Hasan, Phys. Rev. Lett. 103, 146401 (2009).

[9] Y.-L. Chen, J. G. Analytis, J.-H. Chu, Z.-K. Liu, S.-K. Mo, X.-L. Qi, H.-J. Zhang, D.-H. Lu, X. Dai, Z. Fang, S.-C. Zhang, I. R. Fisher, Z. Hussain, Z.-X. Shen, Science 325, 178 (2009).

[10] Y. Xia, D. Qian, D. Hsieh, L. Wray, A. Pal, H. Lin, A. Bansil, D. Grauer, Y. S. Hor, R. J. Cava, and M. Z. Hasan, Nat. Phys. 5, 398 (2009).

[11] P. Cheng, C. Song, T. Zhang, Y. Zhang, Y. Wang, J.-F. Jia, J. Wang, Y. Wang, B.-F. Zhu, X. Chen, X. Ma, K. He, L. Wang, X. Dai, Z. Fang, X. C. Xie, X. L. Q, C. X. Liu, S. C. Zhang, and Q. K. Xue, Phys. Rev. Lett. 105, 076801 (2010).

[12] L. Fu and C. L. Kane, Phys. Rev. Lett. 100, 096407 (2008).

[13] X.-L. Qi, T. L. Hughes, and S.-C. Zhang, Phys. Rev. B 82, 184516 (2010).

[14] L. Fu and C. L. kane, Phys. Rev. B 79, 161408 (2009).

[15] Y. Tanaka, T. Yokoyama, and N. nagaosa, Phys. Rev. Lett. 103, 107002 (2009).

[16] R. Lutchyn, J. Sau, and S. Das Sarma, Phys. Rev. lett. 105, 077001 (2010).

[17] A. R. Akhmerov, J. Nilsson, and C. W. J. Beenakker, Phys. Rev. Leet. 1029216404 (2009).

[18] L. Fu and C. L. Kane, Phys. Rev. lett. 102, 216403 (2009).

[19] J. Linder, Y. Tanaka, T. Yokoyama, A. Sudbø, and N. nagaosa, Phys. Rev. Lett. 104, 067001 (2010).

[20] S. B. Chung, X.-L. Qi, J. Maciejko, and S.-C. Zhang, Phys. Rev. B 83, 100512(R) (2011).

[21] R. Shindou, A. Furusaki, and N. Nagaosa, Phys. Rev. B 82, 180505(R) (2010).

[22] M. I. Salkola, A. V. Balatsky, and J. R. Schrieffer, Phys. Re.v B 55, 12648 (1997).

[23] Q. Liu, C.-X. Liu, C. Xu, X.-L. Qi, and S.-C. Zhang, Phys. Rev. Lett. 102, 156603 (2009).

[24] S. Sasaki, M. Kriener, K. Segawa, K. Yada, Y. Tanaka, M. Sato, and Y. Ando, arXiv:1108.1101v1 (2011).

[25] D. K. Morr and N. A. Stavropoulos, Phys. Rev. B 67, 020502(R) (2003); Phys. Rev. Lett. 92, 107006 (2004); N. A. Stavropoulos and D. K. Morr, Phys. Rev. B 71, 14050(R) (2005). 
[26] A. V. Balatsky, I. Vekhter, J.-X. Zhu, Rev. Mod. Phys. 78, 373 (2006). 